\title{
RESULTS OF RADICAL SURGICAL TREATMENT OF CHOLANGIOCARCINOMAS
}

\author{
Valeriy Boyko \\ Surgery Department No. 1 \\ Kharkiv National Medical University \\ 4 Nauki ave., Kharkiv, Ukraine, 61022 \\ Government Institution "V. T. Zaitsev Institute of General and Urgent Surgery of the National \\ Academy of Medical Sciences of Ukraine" \\ 1 Balakireva entry, Kharkiv, Ukraine, 61018 \\ Yuriy Avdosyev \\ Department of Intervention Radiology \\ Government Institution "V. T. Zaitsev Institute of General and Urgent Surgery of the National \\ Academy of Medical Sciences of Ukraine" \\ 1 Balakireva entry, Kharkiv, Ukraine, 61018
}

\author{
Anastasiia Sochnieva \\ Department of Surgery No. 1 \\ Kharkiv National Medical University \\ 4 Nauki ave., Kharkiv, Ukraine, 61022
}

\begin{abstract}
Radical operations of cholangiocarcinomas are connected with the great number of post-operative complications, and lethality reaches $15-30 \%$. The most debatable questions remain the choice of the operation type depending on localization and spreading of a tumor and also the expedience of using mini-invasive technologies as a preoperational preparation to the radical surgical treatment. We would like to share the little experience of the radical surgical treatment of cholangiocarcinomas.

The research aim is to analyze results of radical surgical treatment of patients with cholangiocarcinoma.

Matherials and methods. We have analyzed the outcomes of surgical treatment in 18 patients with cholangiocarcinomas. Tumor localization was determined according to the Bismuth-Corlett classification. Type 1 tumors were found in 2 (11.1 \%), type 2 in $4(22.2 \%)$, type 3A in $5(27.8 \%)$, type 3B in $4(22.2 \%)$, type 4 in $1(5.6 \%)$, and distal localization in $2(11.1 \%)$ patients. As biliary decompression, 9 (50\%) patients underwent percutaneous transhepatic biliary duct drainage (PTBD), and another 9 (50\%) patients were operated without preoperative biliary decompression Pre-operative embolization of portal vein branches with the aim to increase the low volume of liver after anticipated resection was performed in 4 (22.2\%) patients.

Results. After the embolization of portal vein branches, the estimated residual volume of hepatic parenchyma increased from $33.4 \%$ to $45.7 \%$. Patients with cholagiocarcinomas of different localization performed the radical operations: isolated hepaticocholedochus resection in $5(27.8 \%)$ patients, hepaticocholedochus resection combined with Taj Mahal hepatic resection in 1 (5.6\%), right hemihepatectomy in 5 (27.8\%), left hemihepatectomy in 4 (22.2\%), extended right hemihepatectomy in 1 (5.6\%), and pancreoduodenal resection in $2(11.1 \%)$ patients. Complications of radical surgeries were observed in 4 (22.2\%) patients. Lethal outcomes occurred in $3(16.7 \%)$ patients.

Conclusions. Radical operations are attended with complications in $22.2 \%$ and lethality in $16.7 \%$. As a preoperative preparation it is possible to use mini-invasive interventions for the biliary decompression and increase of hepatic parenchyma volume.

Keywords: cholangiocarcinoma, mechanical jaundice, radical surgery, preoperative biliary decompression.
\end{abstract}

\section{Introduction}

Cholangiocarcinoma is a severe malign bile duct condition occurring in $3 \%$ of all cancer types $[1,2]$. Its main symptom is mechanical jaundice. In $90 \%$ of patients, it indicated non-resectability of the tumor process [3-5].

A radical surgery is the only way to recovery. Radicalism of the operative treatment can be attained only executing a wide resection of biliary channels and gallbladder, elimination of paracholedochal cellulose and lymph nodes, and in several cases the wide resection of a liver and 
pancreaticoduodeal resection [6,7]. Surgical interventions in the presence of mechanical jaundice and infectious and inflammatory complications relate to a large number of post-operative complications and a high mortality rate [8].

In the epoch of mini-invasive interventions for the decompression of bile ducts, the injury rate during the latter has somewhat decreased. However, the appropriateness of mini-invasive interventions for the preparation of patients for radical surgeries still remains a topical issue.

\section{Aim of research}

Analyze results of radical surgical treatment of patients with cholangiocarcinoma.

\section{Materials and methods}

We have analyzed results of 18 radical surgeries, performed in patients with cholangiocarcinoma. The patients' age varied from 30 to 72 years averaging 63.4 years. There were 8 males (44.4\%) and 10 females 21 (55.6\%). Tumor localization was determined according to the Bismuth-Corlett classification. Type 1 tumors were found in $2(11.1 \%)$, type 2 in $4(22.2 \%)$, type 3A in $5(27.8 \%)$, type $3 \mathrm{~B}$ in $4(22.2 \%)$, type 4 in $1(5.6 \%)$, and distal localization in $2(11.1 \%)$ patients. Cholestasis duration was assessed according to Y. V. Smirnov's classification. The main indicator of mechanical jaundice severity was considered as the level of general bilirubin of blood serum that was increased mainly at the expanse of the direct fraction. The level of general bilirubin of blood serum was determined by the standard Endrashik method in the biochemical laboratory of SI "Institute of general and urgent surgery, named after V. T. Zaitsev, NAMS of Ukraine". The clinical diagnosis was established using ultrasound tests, spiral computer tomography, magnetic resonance tomography, and percutaneous transhepatic cholangiography. As biliary decompression, 9 (50 \%) patients underwent percutaneous transhepatic biliary duct drainage (PTBD), and another 9 (50\%) patients were operated without preoperative biliary decompression Pre-operative embolization of portal vein branches with the aim to increase the low volume of liver after anticipated resection was performed in 4 (22.2\%) patients. All patients further underwent radical surgeries with lymph node dissection of the hepatoduodenal ligament.

\section{Results}

Blood serum bilirubin concentration in patients at admission varied from 60 to $502 \mu \mathrm{mole} / 1$ with the median value of $307.5 \mu$ mole/l. Mean bilirubin was $328.17 \pm 108.79 \mu$ mole $/ 1$.

For the purpose of biliary decompression, 9 (50\%) patients underwent percutaneous transhepatic biliary duct drainage (PTBD).

On Fig. 1, 2 are presented cholangiograms of patients with cholangiocarcinomas of II, IIIA and IV types, who underwent PTBD before radical operations.

Decompression lasted from 5 to 32 days, with a median of 13 days and a mean of 13.17 \pm 6.76 days. Complications after the drainage stage were observed in $5(27.8 \%)$ patients. The median level of blood serum bilirubin at the radical surgery was $109.5 \mu \mathrm{mole} / 1$. Complications were as follows: migration of biliary drainage in 1 (5.6 \%) cases, hemobilia in 1 (5.6 \%), biloma formation in $1(5.6 \%)$, development of cholangitis in 1(5.6\%), and hepatic failure in $1(5.6 \%)$ case. There were no lethal outcomes following the biliary decompression.

After the embolization of portal vein branches, the estimated residual volume of hepatic parenchyma increased from $33.4 \%$ to $45.7 \%$.

The second stage included the following radical operations: isolated hepaticocholedochus resection in $5(27.8 \%)$ patients, hepaticocholedochus resection combined with Taj Mahal hepatic resection in 1 (5.6\%), right hemihepatectomy in $5(27.8 \%)$, left hemihepatectomy in $4(22.2 \%)$, extended right hemihepatectomy in 1 (5.6\%), and pancreoduodenal resection in $2(11.1 \%)$ patients. All large hepatic resections were combined with the total caudal hemihepatectomy and hepaticocholedochus resection.

Complications of radical surgeries were observed in 4 (22.2\%) patients. Many of them demonstrated several complications at once. Hepatic failure occurred in 2 (11.1\%) patients. Purulent cholangitis was observed in $1(5.6 \%$ ) patient. Hepaticojejunostomy failure occurred in $15.6 \%$ ) 
patients. In this patient, biloma formed in the subhepatic region required surgical treatment, so percutaneous transhepatic puncture and biloma drainage were performed under the control of X-ray visualization. Lethal outcomes occurred in 3 (16.7 \%) patients. In 1 (5.6\%) case, the death was caused by multisystemic failure developed at the background of purulent cholangitis. Fulminant hepatic failure caused by the low hepatic volume developed in $1(5.6 \%)$ patient. $1(5.6 \%)$ death was caused by erosive bleeding from the hepatic artery.

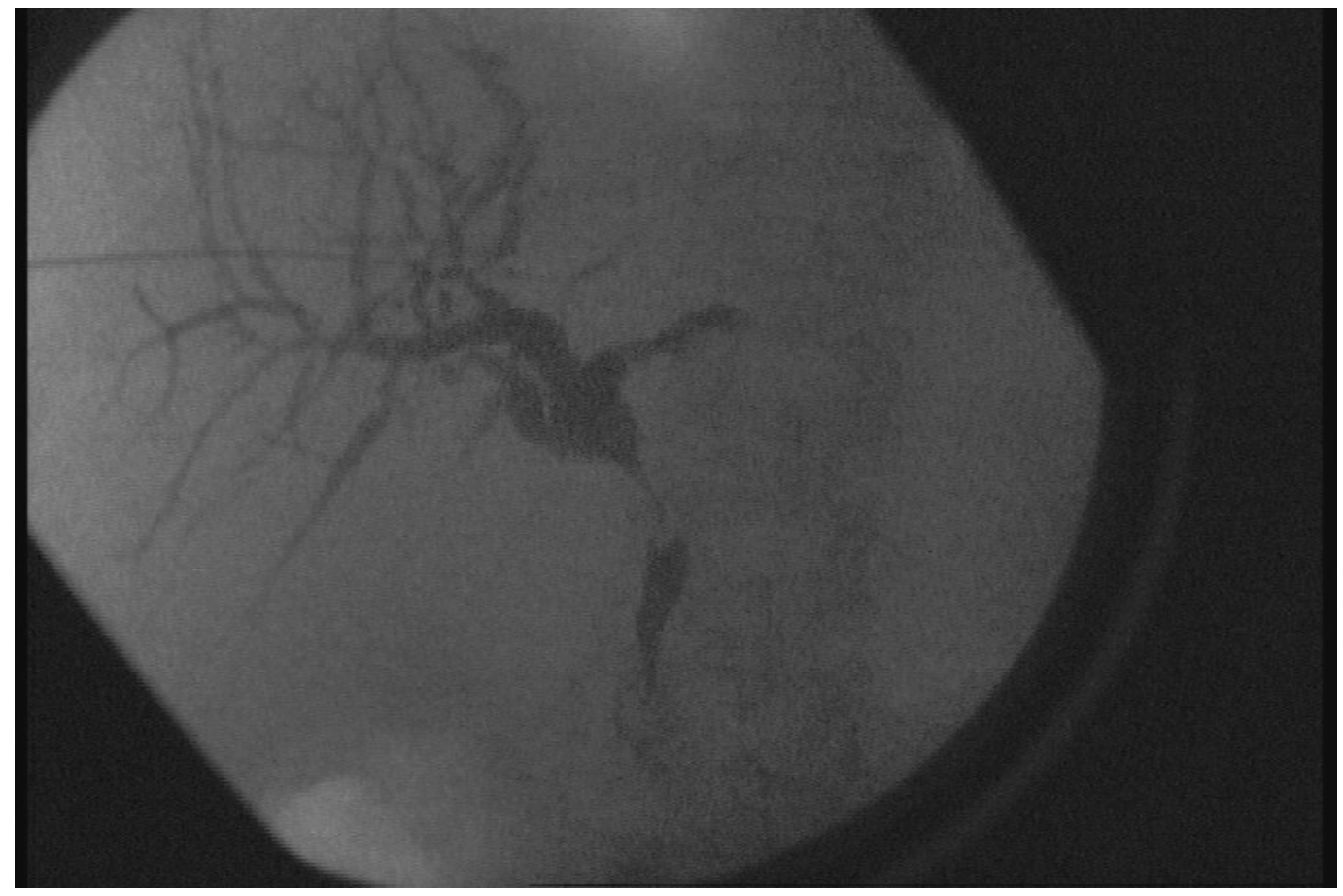

Fig. 1. Patient with cholagiocarcinoma of II type

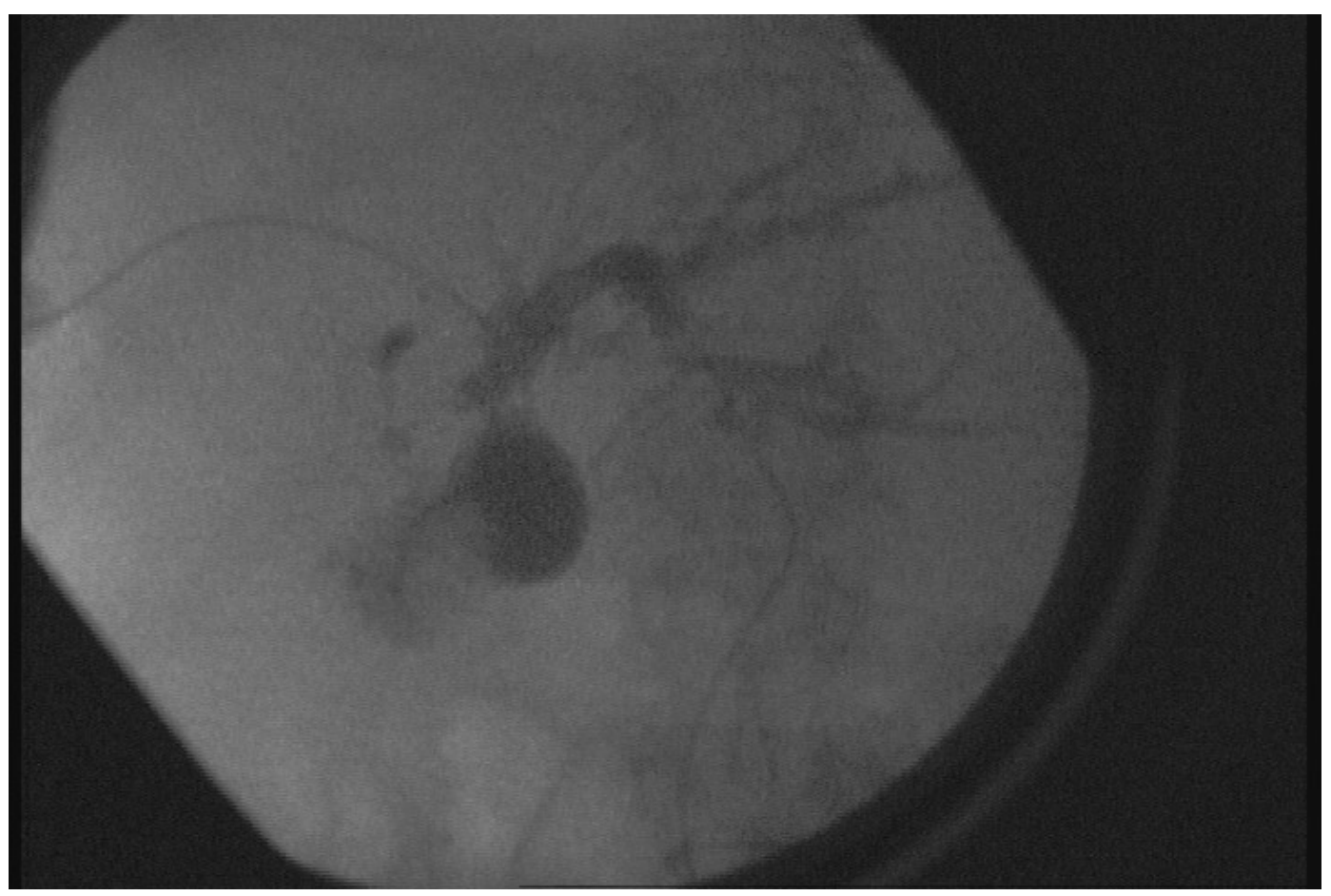

Fig. 2. Patient with cholagiocarcinoma of III A type 


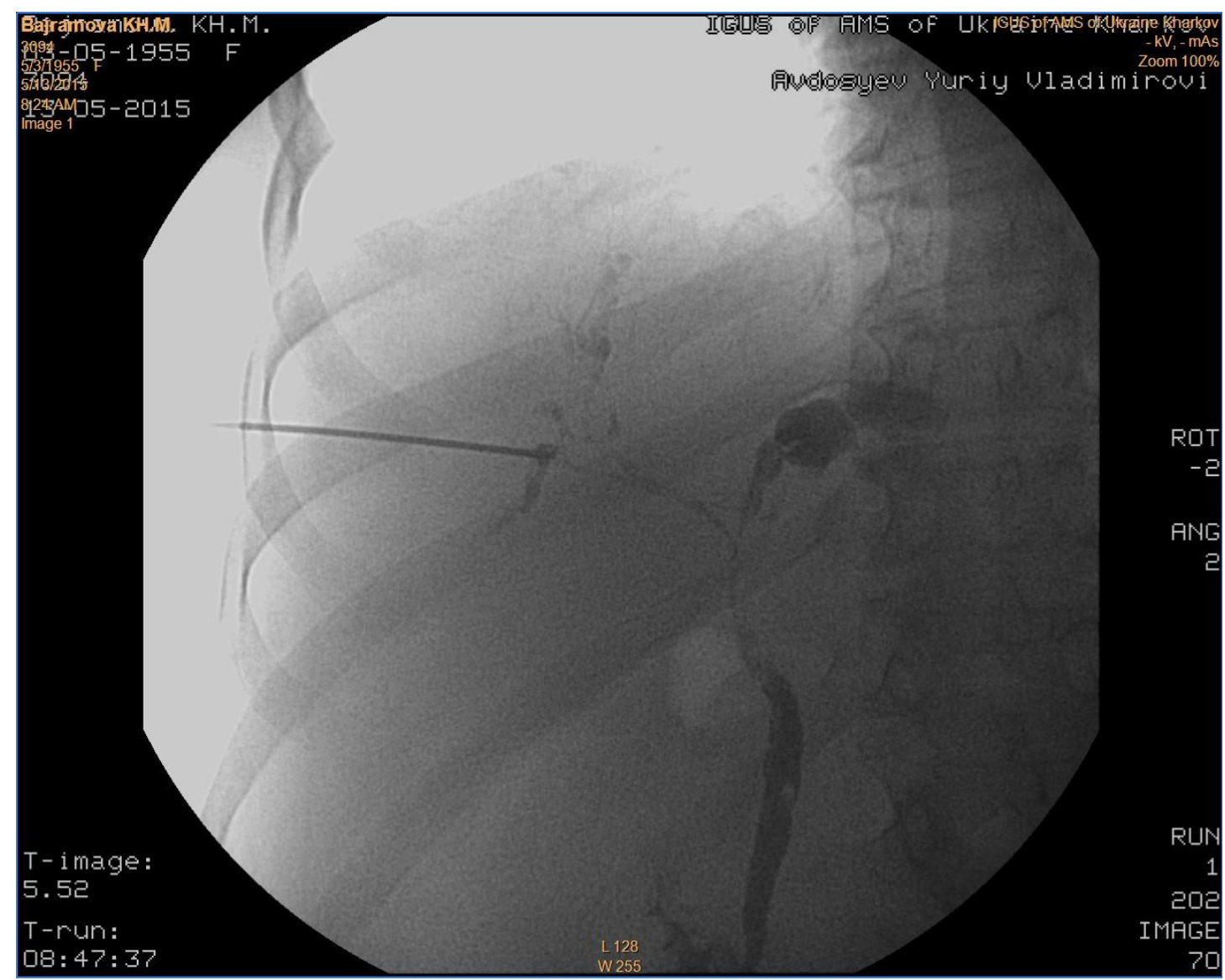

Fig. 3. Patient with cholagiocarcinoma of IV type

\section{Discussion}

Biliary decompression was performed using percutaneous transhepatic biliary duct drainage. If possible, biliary duct drainage should be performed on the hepatic lobe remaining after resection. Separate drainage of lobar hepatic ducts is performed if they are branched on the subsegmentary level, in the cases of cholangitis, cholangic liver abscesses, and low rates of hyperbilirubinemia reduction.

It is now assumed that Type 3 and 4 tumors according to the Bismuth-Corlette classification are not contraindications against radical surgical treatment [9]. Tumor invasion of hepatoduodenal ligament vessels is not a contraindication against a radical surgery, either. Portal vein resection allows reaching $\mathrm{R}-0$ resection margins and does not worsen the operative treatment prognosis [10]. It can be concluded based on our study that, in a case of adequate and timely mini-invasive interventions as preparation of patients for radical surgeries, all types of tumors are radically curable in the absence of tumor invasion in the hepatic artery and in the absence of remote metastases $[6,7,11-13]$.

According to our observations, all tumor types are operable at the adequate use of mini-invasive interventions as a preparation of a patient to the radical operation, at the absence of a tumor invasion in the hepatic artery and remote metastases.

We have realized $9(50 \%)$ radical operations at IIIa and IIIb types of cholagiocarcinoma. The resection volume was no less than the hemihepatectomy, correspondent to the side of lobar channel injury with the total caudal lobectomy. The expanded right-side hemihepatectomy with the total caudal lobectomy was executed in $11(5.6 \%)$ patients at IV type of cholangiocarcinoma. The resection of the portal vein was executed in $3(16.7 \%)$ patients. At I and II tumor types were 
realized $6(33.3 \%)$ isolated resections of bile-excreting tracts with the reconstructive stage - hepatico- and bihepatocojejuanamastosis by Roux. Combined resections of a liver, combined with the total caudal lobectomy and resection of extra-hepatic biliary channels are executed by most world hepatobiliary surgical centers [14-17].

At cholangiocarcinoma localization in distal parts of hepaticocholedochus we consider the adequate volume of the surgical treatment as a pancreatoduodenal resection execution. The last one was executed in $2(11.1 \%)$ patients.

On Fig. 4 is presented the reconstructive stage after the right-side hemihepatectomy - hepaticojejunostomy by Roux with the left hepatic channel, keeping PTBD.

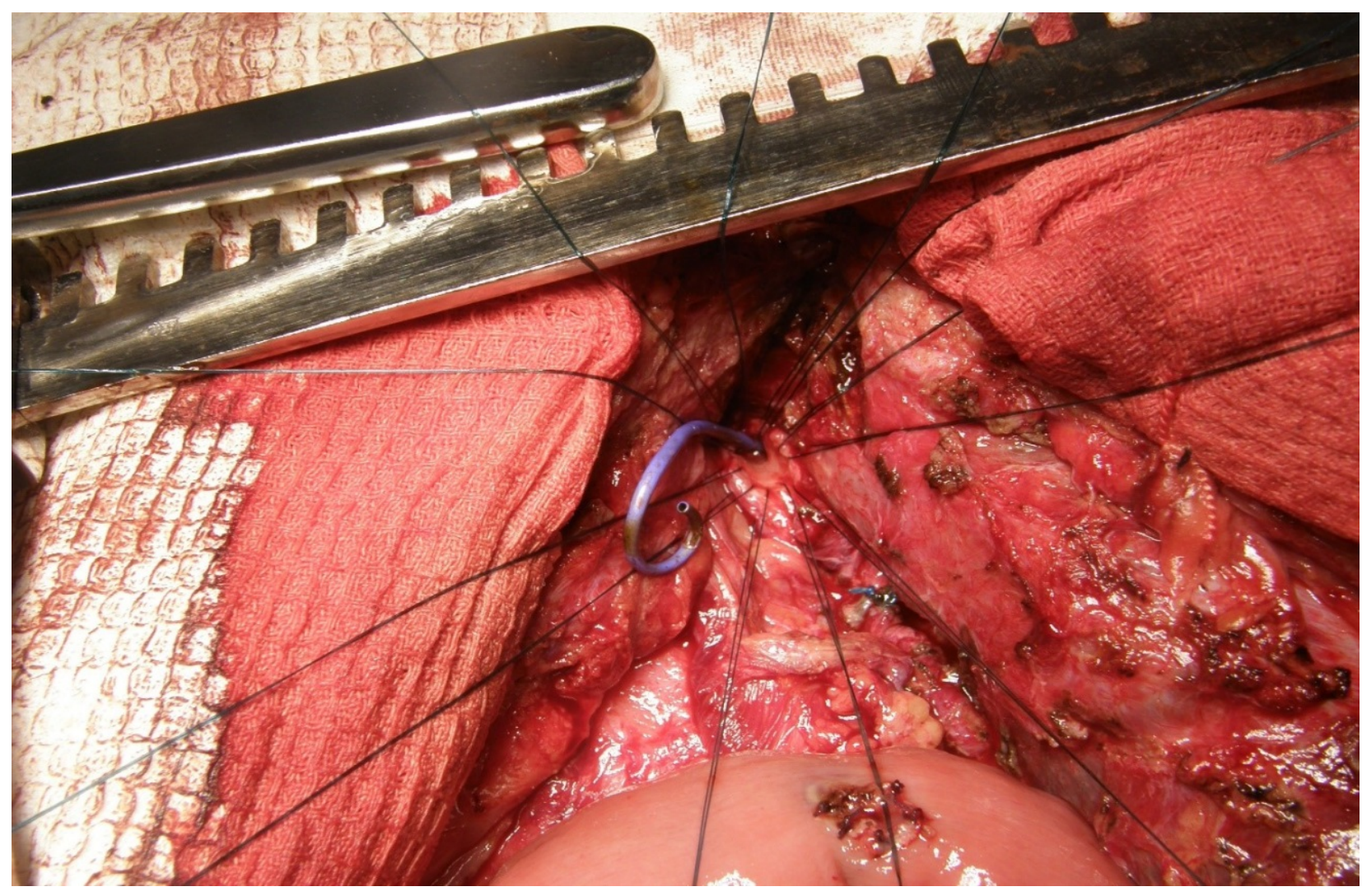

Fig. 4. Hepaticojejunostomy by Roux with the left hepatic channel, keeping PTBD

On Fig. 5 is presented the final look of the operation after the right-side hemihepatoectomy, executed as to cholanocarcinoma of IIIA type.

Radical surgeries are usually accompanied by numerous post-operative complications and high mortality rates, and this is despite the improvement in direct outcomes of surgical treatment [18]. Literature refers to the $13 \%$ mortality rate and complications in more than $60 \%$ of patients [19]. We observed complications after radical operations in $4(22.2 \%)$ patients. They were overcome by conservative and mini-invasive methods.

We think that the special attention must be paid to the preoperative preparation. The operative preparation of patients to such essential surgical interventions is widely discussed in surgical communities. Murakami Y. And co-authors [20] recommend to realize the biliary decompression to all patients with mechanical jaundice.

At the prognosis of the resection of the great volume of a liver $4(10.3 \%)$ patients underwent the embolization of portal vein branches. The supposed volume of parenchyma of a liver increased in average from 33.4 to $45.7 \%$. Such tactics of patients' treatment is observed by most Japan hepatobiliary surgeons [20]. 


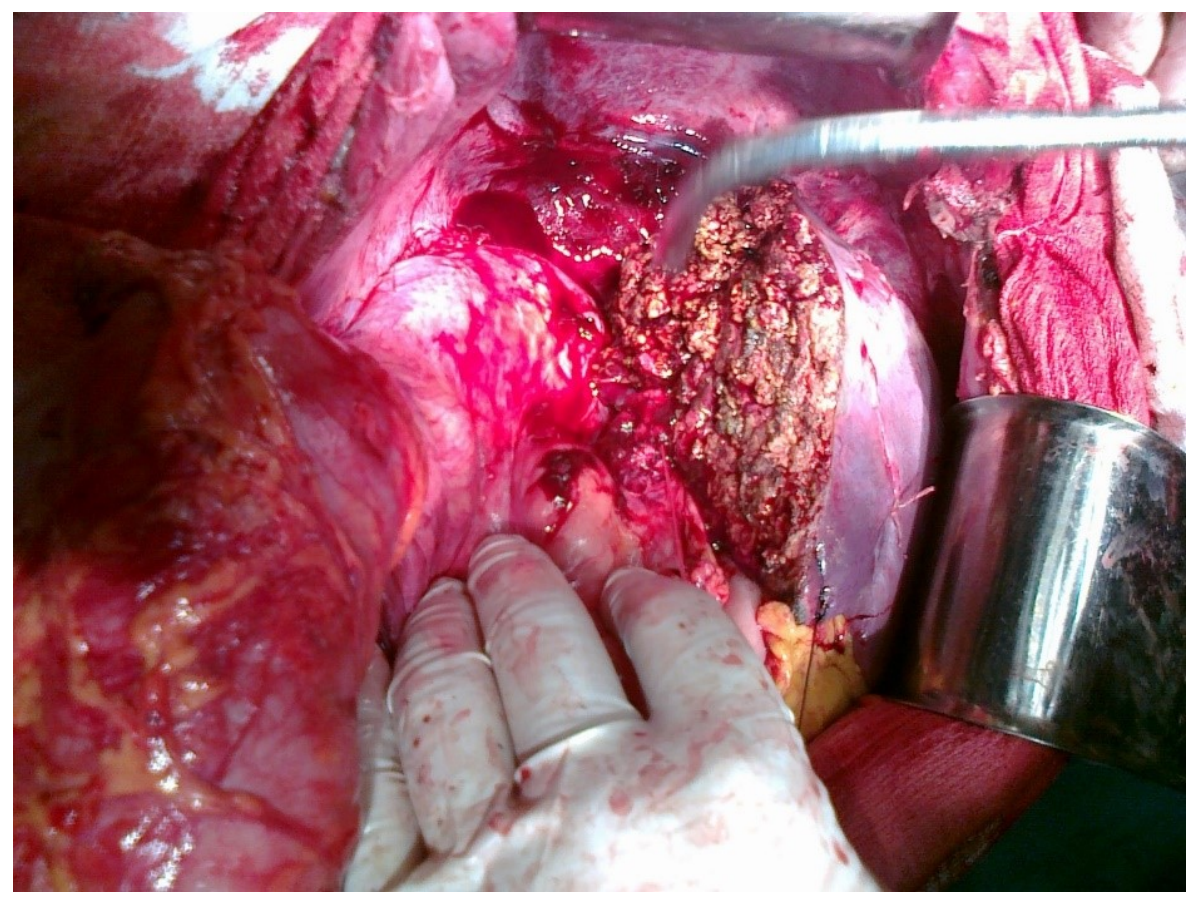

Fig. 5. Operational zone after the right-side hemihepatoectomy, executed as to cholanocarcinoma of IIIA type

We agree with the aforesaid authors and observe their recommendations in our treatment tactics. We execute the biliary decompression by the transcutaneous transhepatic cholangiodrainage. It is wanted to execute the cholangiodrainage of the last part of a liver. The separate drainage of lobar channels we execute at their separation at subsegmentary level, at presence of cholangitis and cholanogiogenic abscesses of a liver and also at the low speed of the hyperbilirubidemia decrease. We realized the embolization of portal vein branches at the predicted volume of the liver resection more than 60-70 \%. After realizing the embolization of the portal vein branches, all patients demonstrated the increase of the remained liver in volume of 2-3 segments. 1 (5.6\%) patient, who underwent the embolization of portal vein branches, suffered in the postoperative period from hepatic failure that was not stopped at the background of the intensive conservative therapy. At the preserved hepatic function and volume of remained parenchyma more than 30-40\% we don't consider it necessary to realize the embolization of portal vein branches. In our study was established that the risk of the liver small lobar syndrome development exists.

\section{Conclusions}

1. The complication rate of biliary decompression with mini-invasive drainage is $27.8 \%$. The embolization of portal vein branches prevent hepatic failure caused by the low hepatic volume. Pre-operative biliary decompression allows a patient to be prepared for radical surgical treatment.

2. Complications at the radical treatment stage were observed in $22.2 \%$ of patients. The mortality rate in patients, who underwent radical surgeries, is $16.7 \%$. Hepatic insufficiency after the radical stage of surgical treatment is the main cause of development of postoperative complications, as well as a high level of lethality.

\section{References}

[1] Malhi, H., Gores, G. J. (2006). Cholangiocarcinoma: Modern advances in understanding a deadly old disease. Journal of Hepatology, 45 (6), 856-867. doi: 10.1016/j.jhep.2006.09.001

[2] Kawasaki, S., Imamura, H., Kobayashi, A., Noike, T., Miwa, S., Miyagawa, S. (2003). Results of Surgical Resection for Patients With Hilar Bile Duct Cancer. Annals of Surgery, 238 (1), 84-92. doi: 10.1097/01.sla.0000074984.83031.02 
[3] Chen, X.-P., Lau, W. Y., Huang, Z.-Y., Zhang, Z.-W., Chen, Y.-F., Zhang, W.-G., Qiu, F.-Z. (2009). Extent of liver resection for hilar cholangiocarcinoma. British Journal of Surgery, 96 (10), 1167-1175. doi: $10.1002 /$ bjs. 6618

[4] Ferrero, A., Lo Tesoriere, R., Viganò, L., Caggiano, L., Sgotto, E., Capussotti, L. (2008). Preoperative Biliary Drainage Increases Infectious Complications after Hepatectomy for Proximal Bile Duct Tumor Obstruction. World Journal of Surgery, 33 (2), 318-325. doi: 10.1007/s00268-008-9830-3

[5] Kuang, D., Wang, G.-P. (2010). Hilar cholangiocarcinoma: Pathology and tumor biology. Frontiers of Medicine in China, 4 (4), 371-377. doi: 10.1007/s11684-010-0130-6

[6] Boyko, V. V., Maloshtan, A. V. (2008). Clinical-anatomic substantiation of radical surgery for cancer of the extrahepatic biliary tract. Kharkiv surgical school, 4, 105-113.

[7] Dotsenko, A. P., Zinchenko, E. I. (1977). Cancer of the gallbladder and extrahepatic biliary tract. Moscow: Medicine, 312.

[8] Murakami, Y., Uemura, K., Sudo, T., Hashimoto, Y., Nakashima, A., Kondo, N. et. al. (2010). Prognostic Factors After Surgical Resection for Intrahepatic, Hilar, and Distal Cholangiocarcinoma. Annals of Surgical Oncology, 18 (3), 651-658. doi: 10.1245/s10434-010-1325-4

[9] Tompkins, R. K., Saunders, K. D., Roslin, J. J. et al. (1990). Changing patterns in diagnosis and management of bile duct cancer. Annals of Surgery, 211, 611.

[10] Igami, T., Nishio, H., Ebata, T., Yokoyama, Y., Sugawara, G., Nimura, Y., Nagino, M. (2009). Surgical treatment of hilar cholangiocarcinoma in the "new era": the Nagoya University experience. Journal of Hepato-Biliary-Pancreatic Sciences, 17 (4), 449-454. doi: 10.1007/s00534-009-0209-0

[11] Neuhaus, P., Jonas, S., Bechstein, W. O., Lohmann, R., Radke, C., Kling, N. et. al. (1999). Extended Resections for Hilar Cholangiocarcinoma. Annals of Surgery, 230 (6), 808-818. doi: 10.1097/00000658199912000-00010

[12] Shalimov, A. A., Kopchak, V. M. Dronov, A. I. et. al. (2001). The clinic, diagnosis and treatment of tumors of the extrahepatic bile ducts. Clinical surgery, 6, 11-14.

[13] Nazyrov, F. G., Ikramov, A. I., Akbar, M. M. et. al. (2010). Diagnosis and tactics of surgical treatment of patients with tumors of the proximal bile duct. Surgery, 6, 36-39.

[14] Nathan, H., Pawlik, T. M., Wolfgang, C. L., Choti, M. A., Cameron, J. L., Schulick, R. D. (2007). Trends in Survival after Surgery for Cholangiocarcinoma: A 30-Year Population-Based SEER Database Analysis. Journal of Gastrointestinal Surgery, 11 (11), 1488-1497. doi: 10.1007/s11605-007-0282-0

[15] Que, F. G., Nagorney, D. M. (1994). Resection of "recurrent" colorectal metastases to the liver. British Journal of Surgery, 81 (2), 255-258. doi: 10.1002/bjs.1800810234

[16] Timm, S., Gassel, H.-J., Thiede, A. (2007). Die chirurgische Therapie proximaler extrahepatischer Gallengangstumoren (Klatskin-Tumoren). Zentralblatt Für Chirurgie, 132 (4), 328-335. doi: 10.1055/ s-2007-981201

[17] Seyama, Y., Kubota, K., Sano, K., Noie, T., Takayama, T., Kosuge, T., Makuuchi, M. (2003). Long-Term Outcome of Extended Hemihepatectomy for Hilar Bile Duct Cancer With No Mortality and High Survival Rate. Annals of Surgery, 238 (1), 73-83. doi: 10.1097/01.sla.0000074960.55004.72

[18] Nimura, Y., Kamiya, J., Kondo, S., Nagino, M., Uesaka, K., Oda, K. et. al. (2000). Aggressive preoperative management and extended surgery for hilar cholangiocarcinoma: Nagoya experience. Journal of Hepato-Biliary-Pancreatic Surgery, 7 (2), 155-162. doi: 10.1007/s005340050170

[19] Nagino, M., Kamiya, J., Arai, T., Nishio, H., Ebata, T., Nimura, Y. (2006). «Anatomic» Right Hepatic Trisectionectomy (Extended Right Hepatectomy) With Caudate Lobectomy for Hilar Cholangiocarcinoma. Annals of Surgery, 243 (1), 28-32. doi: 10.1097/01.sla.0000193604.72436.63

[20] Vishnevsky, V. A., Tarasyuk, T. I., Ikramov, R. Z. (2005). Radical surgery for cancer of the proximal bile duct. Annals of surgical hepatology, 10 (3), 35-42. 